\title{
Note on results from the experiments
}

This appendix provides a summary of the results from our experiments. Differences in the unit of analysis and study design prevent a uniform measure of effect size, which may be comparable across the interventions. However, these figures summarize the percentage shifts the intervention achieved or give a qualitative assessment in the case of the design experiments.

Table A1.1 Effects of the experiments

\begin{tabular}{|l|l|l|l|}
\hline Chapter \& topic & Intervention & $\begin{array}{l}\text { Effect of the } \\
\text { intervention in } \\
\text { comparison to a } \\
\text { control group }\end{array}$ & $\begin{array}{l}\text { Nudge } \\
\text { or think }\end{array}$ \\
\hline $\begin{array}{l}\text { Chapter 3 } \\
\text { Recycling } \\
\text { experiment 1 } \\
\text { (household } \\
\text { recycling) }\end{array}$ & Canvassing & $\begin{array}{l}5 \% \text { increase in } \\
\text { recycling } \\
\text { (immediate); drops } \\
\text { to 4\% increase (3 } \\
\text { months later) }\end{array}$ & Nudge \\
\hline $\begin{array}{l}\text { Recycling } \\
\text { experiment 2 } \\
\text { (food waste) }\end{array}$ & Feedback & $\begin{array}{l}3 \% \text { increase in } \\
\text { recycling }\end{array}$ & Nudge \\
\hline
\end{tabular}


Note on results from the experiments

\begin{tabular}{|c|c|c|c|}
\hline $\begin{array}{l}\text { Chapter } 4 \\
\text { Volunteering }\end{array}$ & Asking & $\begin{array}{l}\text { Initial surge of } \\
\text { interest but no } \\
\text { change in } \\
\text { volunteering levels }\end{array}$ & Nudge \\
\hline $\begin{array}{l}\text { Chapter } 5 \\
\text { Voting }\end{array}$ & $\begin{array}{l}\text { i) Telephone } \\
\text { calls } \\
\text { ii) Canvassing }\end{array}$ & $\begin{array}{l}\text { i) } 3.5 \% \text { more votes } \\
\text { ii) } 3.6 \% \text { more votes }\end{array}$ & Nudge \\
\hline $\begin{array}{l}\text { Chapter } 6 \\
\text { Petitioning }\end{array}$ & $\begin{array}{l}\text { Social } \\
\text { information }\end{array}$ & $\begin{array}{l}\text { i) } 9 \% \text { more } \\
\text { petitions signed in } \\
\text { lab study } \\
\text { ii) } 5.2 \% \text { more } \\
\text { petitions signed in } \\
\text { field study }\end{array}$ & Nudge \\
\hline $\begin{array}{l}\text { Chapter } 7 \\
\text { Giving (books) }\end{array}$ & $\begin{array}{l}\text { i) Pledging } \\
\text { ii) Pledging \& } \\
\text { publicity }\end{array}$ & $\begin{array}{l}\text { i) } 0.9 \% \text { more } \\
\text { donations } \\
\text { (non-significant) } \\
\text { ii) } 1.6 \% \text { more } \\
\text { donations }\end{array}$ & Nudge \\
\hline $\begin{array}{l}\text { Chapter } 8 \\
\text { Organ donating } \\
\text { experiment } 1 \\
\text { (information \& } \\
\text { discussion) }\end{array}$ & $\begin{array}{l}\text { i) Information } \\
\text { ii) Information } \\
\text { \& discussion }\end{array}$ & $\begin{array}{l}\text { i) } 4 \% \text { increase in } \\
\text { registrations } \\
\text { ii) } 15 \% \text { decrease in } \\
\text { registrations }\end{array}$ & $\begin{array}{l}\text { Nudge } \\
\text { v. } \\
\text { Nudge } \\
\text { \& think }\end{array}$ \\
\hline $\begin{array}{l}\text { Organ donating } \\
\text { experiment } 2 \\
\text { (choice } \\
\text { architecture) }\end{array}$ & $\begin{array}{l}\text { i) Presumed } \\
\text { consent } \\
\text { ii) Mandated } \\
\text { choice }\end{array}$ & $\begin{array}{l}\text { i) } 8 \% \text { increase in } \\
\text { registrations } \\
\text { ii) } 5 \% \text { increase in } \\
\text { registrations }\end{array}$ & $\begin{array}{l}\text { Nudge } \\
\text { \& think }\end{array}$ \\
\hline $\begin{array}{l}\text { Chapter } 9 \\
\text { Debating (online } \\
\text { experiment) }\end{array}$ & $\begin{array}{l}\text { i) Discussion } \\
\text { forums \& } \\
\text { information } \\
\text { ii) Information } \\
\text { only }\end{array}$ & $\begin{array}{l}\text { i) Modest opinion } \\
\text { shifts in policy } \\
\text { preferences; no } \\
\text { changes in policy } \\
\text { knowledge } \\
\text { ii) No change }\end{array}$ & Think \\
\hline
\end{tabular}


Note on results from the experiments

\begin{tabular}{|l|l|l|l|}
\hline $\begin{array}{l}\text { Chapter 10 } \\
\text { Including (DVD) }\end{array}$ & $\begin{array}{l}\text { DVD portraying } \\
\text { views of } \\
\text { politically } \\
\text { disengaged } \\
\text { groups }\end{array}$ & $\begin{array}{l}\text { Discussions with } \\
\text { DVD generated } \\
\text { debate, where } \\
\text { decision-makers } \\
\text { were more } \\
\text { considerate of } \\
\text { disengaged groups; } \\
\text { DVDs influenced } \\
\text { Area Boards' } \\
\text { thinking about } \\
\text { future engagement } \\
\text { but created some } \\
\text { resistance amongst } \\
\text { councillors }\end{array}$ & \\
& & $\begin{array}{l}\text { i) 21\% response } \\
\text { from councillors } \\
\text { ii) 16\% response } \\
\text { from councillors } \\
\text { (no control group } \\
\text { in this study) }\end{array}$ & \\
\hline Chapter 11 & i) Weak letter & \\
Linking (letters to \\
councillors) & ii) Strong letter & \\
& & &
\end{tabular}

